\title{
Fatty Acid Synthase Inhibition by Amentoflavone Induces Apoptosis and Antiproliferation in Human Breast Cancer Cells
}

\author{
Jin Sun LeE, ${ }^{a, \#}$ Myung Sun LeE, ${ }^{b, \#}$ Won Keun OH, ${ }^{c}$ and Ji Young SuL ${ }^{*, a, d}$ \\ ${ }^{a}$ Department of Surgery, Chungnam National University Hospital; 33 Munhwa-ro, Jung-gu, Daejeon 301-721, Korea: \\ ${ }^{b}$ Department of Food and Nutrition, Chungnam National University; 220 Gung-dong, Yuseong-gu, Daejeon 305-764, \\ Korea: ${ }^{c}$ College of Pharmacy, Chosun University; 375 Seosuk-dong, Dong-gu, Gwangju 501-759, Korea: and \\ ${ }^{d}$ Department of Surgery and Research Institute for Medical Science, Chungnam National University School of Medicine; 6 \\ Munhwa-dong, Jung-gu, Daejeon 301-130, Korea. Received October 8, 2008; accepted January 23, 2009
}

Fatty acid synthase (FASN) is highly expressed in breast carcinomas to support their continuous growth and proliferation, but has low expression level in normal tissues. Considerable interest has been developed in searching for novel FASN inhibitors as a therapeutic target for breast cancer. In present study, amentoflavone was isolated from Selaginella tamariscina, a traditional oriental medicine that has been used to treat cancer for many years, and was found to significantly inhibit the in vitro enzymatic activity of FASN at concentrations above $50 \mu$ m. Amentoflavone was also found to decrease fatty acid synthesis by the reduction of $\left[{ }^{3} \mathrm{H}\right]$ acetyl-CoA incorporation into lipids in FASN-overexpressed SK-BR-3 human breast cancer cells. Furthermore, this study showed that amentoflavone, at a concentration greater than $75 \mu \mathrm{M}$, increased the cleavage-activity of caspase-3 and poly (ADP-ribose) polymerase (PARP), and administration of pan-caspase inhibitor Z-VAD-FMK completely rescued the SK-BR-3 cells from PARP cleavages. The sequential internucleosomal DNA fragmentation in SK-BR-3 cells was observed at a concentration of $100 \mu \mathrm{M}$. A decrease in breast cancer cell growth was observed in SK-BR-3 cells at 12 and $24 \mathrm{~h}$ post treatment with $100 \mu \mathrm{m}$ of amentoflavone, followed by a dramatic suppression after $48 \mathrm{~h}$. The inhibition of cancer-growth by amentoflavone was dose-dependent, showing a slight reduction at $50 \mu_{M}$ and significant reduction at concentrations of 75 and $100 \mu \mathrm{M}$. FASN-nonexpressed NIH-3T3 normal cell growth was not decreased by amentoflavone-treatment, both in time- and dose-dependent manners. These data provide evidence that amentoflavone isolated from $S$. tamariscina induced breast cancer apoptosis through blockade of fatty acid synthesis.

Key words amentoflavone; fatty acid synthesis; apoptosis; breast cancer

Fatty acid synthase (FASN), a multifunctional enzyme that catalyzes the terminal steps involved in the synthesis of the 16-carbone fatty acid palmitate from acetyl-CoA, malonyl$\mathrm{CoA}$, and nicotinamide adenine dinucleotide phosphate $(\mathrm{NADPH}),{ }^{1)}$ is highly expressed in a large number of human cancers including breast, colon, ovary, and prostate cancers. ${ }^{2,3)}$ Several reports have shown that the expression of FASN correlates with tumor progression, aggressiveness, and metastasis. ${ }^{45}$ This has led to the investigation of the role of FASN and fatty acid metabolism in cancers becoming a focus for research into the potential diagnosis and treatment of cancer. ${ }^{3)}$ The functional connection between FASN and tumor progression has been provided by the discovery and design of small molecule drugs that inhibit the catalytic activity of FASN ${ }^{6,7)}$ Cerulenin and C75, which target the ketoacyl synthase domain of FASN, were the first small molecules to be described as inhibitors of FASN activity in human tumor cells. ${ }^{3,5}$ Orlistat was described another inhibitor of the thioesterase domain of FASN, which induced cell death in a variety of tumor cell lines and was able to effectively inhibit the growth of tumor xenografts in mice. ${ }^{8,9)}$ Therefore, the strong connection between the activity of FASN and survival of the tumor cell emphasizes the relevance of fatty acid synthesis as an attractive antitumor target.

In breast cancers, a number of studies have pointed toward the potential importance of FASN. For instance, the expression of FASN in malignant breast tumors is associated with a 4-fold increase in risk of death when high levels of FASN expression occurred together with a high proliferative index. $^{10,11)}$ Additionally FASN-inhibitors have shown to de- crease proliferation of cells and induce apoptosis in breast cancer cell lines. ${ }^{12)}$ The treatment of nude mice with FASNinhibitor, C75, showed an inhibition of growth of breast cancer cells without comparable toxicity to normal tissues, ${ }^{13)}$ suggesting that FASN may be a useful target for breast chemotherapy.

Herbal medicines including conventional and complimentary medicines are still prevalent around the world. An increased effort has been invested into the isolation and determination of the molecular structure and mechanisms of bioactive phytochemicals from medicinal plants, which are equally important to lead the pharmaceutical development of synthetic or semi-synthetic drugs. ${ }^{14)}$ Amentoflavone, 8-(5(5,7-dihydroxy-4-oxo-4H-chromen-2-yl)-2-hydroxyphenyl)5,7-dihydroxy-2-(4-hydroxyphenyl)-4H-chromen-4-one, belonging to the biflavonoid class of flavonoids, is abundant in Selaginella tamariscina (Selaginellaceae family) and has been used for the treatment of advanced cancer in traditional oriental medicine. It has also been used as an anti-oxidant, vasorelaxant, anti-HIV and anti-angiogenesis agent. ${ }^{15-18)}$ Nonetheless, there has been no report on the possible effect of amentoflavone on fatty acid metabolism, which plays an important role in cancer cell death.

In the present study, we investigated the possible inhibitory effects of a natural biflavonoid, amentoflavone, on FASN activity in vitro as well as fatty acid synthesis. We subsequently studied the effects of amentoflavone as a fatty acid synthesis-inhibitor on caspase-3-induced apoptosis and growth inhibition using FASN-overexpressed SK-BR3 breast cancer and FASN-nonexpressed NIH-3T3 normal 
cells.

\section{MATERIALS AND METHODS}

Reagents McCOY's 5A Medium, fetal bovine serum (FBS), Dulbecco's modified Eagle's medium (DMEM) and penicillin/streptomycin were obtained from GIBCO/BRL Life Technologies (Grand Island, NY, U.S.A.). Antibody to caspase-3 was purchased from Calbiochem (San Diego, CA, U.S.A.), anti-poly(ADP-ribose)polymerase (PARP) from SantaCruz (Santa Cruz, CA, U.S.A.), and anti-FASN from BD Biosciences (San Jose, CA, U.S.A.). Hoseradish peroxidase-conjugated anti-rabbit and anti-mouse antibodies were from Transduction Lab (Lexington, KY, U.S.A.). Super Signal ${ }^{\circledR}$ West Pico Chemiluminescent substrate was purchased from PIERCE (Rockford, IL, U.S.A.). Cell Counting Kit8 (CCK8) was from Dojindo Laboratories (Kumamoto, Japan). $\left[{ }^{3} \mathrm{H}\right]$ Acetyl-CoA was from PerkinElmer (Waltham, MA, U.S.A.). Nicotinamide adenine dinucleotide phosphate (NADPH), Acetyl-CoA, Malonyl-CoA, Cerulenin, Z-VADFMK and other reagents were obtained from Sigma Chemical Co. (St. Louis, MO, U.S.A.).

Extraction and Purification Amentoflavone was isolated from whole plants of $S$. tamariscina. The dried plant $(2 \mathrm{~kg})$ was extracted with $\mathrm{MeOH}$ at room temperature for 1 week. The $\mathrm{MeOH}$ extract ( $154 \mathrm{~g})$ was resuspended in water and partitioned with EtOAc. The EtOAc fraction (23 g) was subjected to silica gel column chromatography $(10 \times 30 \mathrm{~cm})$ using the mixture of $\mathrm{CHCl}_{3}$ and $\mathrm{MeOH}(90: 1 \rightarrow 0: 1)$ to separate into ten fractions. Fraction \#4 (1 g) was separated by HPLC using an isocratic solvent system of $80 \% \mathrm{MeOH}$ to yield amentoflavone (308 mg). The purity of the compounds was $\geq 95 \%$ as determined by analytical HPLC.

Fatty Acid Synthase Inhibition Assay The preparation, storage and use of FASN from chicken liver were performed as described previously. ${ }^{19)}$ Ammonium sulfate precipitations, gel filtration and anion exchange chromatography were used for purification. FASN has $>95 \%$ purity as estimated from sodium dodecyl sulfate-polyacrylamide gel electrophoresis (SDS-PAGE) with Coomassie blue staining. FASN was incubated with reaction buffer $(200 \mathrm{~mm}$ K-P buffer, $\mathrm{pH} 7.0$, $2.5 \mathrm{~mm}$ DTT, $2.0 \mathrm{~mm}$ EDTA and $1 \mathrm{mg} / \mathrm{ml} \mathrm{BSA}$ ) at $37^{\circ} \mathrm{C}$ for $1 \mathrm{~h}$, and added $0.25 \mathrm{~mm}$ NADPH, $0.4 \mathrm{~nm}$ malonyl-CoA, $0.06 \mathrm{~mm}$ acetyl-CoA, and $0.02 \mu \mathrm{Ci}\left[{ }^{3} \mathrm{H}\right]$ acetyl-CoA, then the reaction was terminated by $60 \% \mathrm{HClO}_{4}$. Fatty acids were extracted with $1 \mathrm{ml}$ of hexane and incorporation of radioactivity into the fatty acids was assessed by scintillation counting.

Cell Culture SK-BR-3 human breast cancer cell line and NIH-3T3 mouse fibroblast were obtained from ATCC (American Type Culture Collection) (Rockville, MD, U.S.A.), and cultured in McCOY's 5A Medium and DMEM, respectively, containing $10 \% \mathrm{FBS}(\mathrm{v} / \mathrm{v})$ and penicillin $(100 \mathrm{U} / \mathrm{ml}) /$ streptomycin $(100 \mu \mathrm{g} / \mathrm{ml})$. Cultures were maintained in humidified incubator at $37^{\circ} \mathrm{C}$ in $5 \% \mathrm{CO}_{2}$.

Fatty Acid Synthesis Activity Inhibition of FASN activity in whole cells was measured by the incorporation of $\left[{ }^{3} \mathrm{H}\right]$ acetyl-CoA into fatty acids. Amentoflavone was exposed to the culture medium for $24 \mathrm{~h}$. After washing out dead cells, adhesive cells were collected and re-plated with the same number, then $\left[{ }^{3} \mathrm{H}\right]$ acetyl-CoA $(2 \mu \mathrm{Ci})$ was added to each well and incubated for $4 \mathrm{~h}$ at $37^{\circ} \mathrm{C}$. Fatty acids were isolated by an initial hypotonic lysis of cells in $50 \mathrm{~mm}$ Tris, $\mathrm{pH} 7.5$, followed by extraction in chloroform: water $(1: 1)$ for $30 \mathrm{~min}$ at room temperature. Lipid phase containing radiolabled lipids was evaporated under $\mathrm{N} 2$ and then counted by scintillation counter.

Western Blotting Analysis Cells were lysed with $10 \mathrm{~mm}$ Tris, pH 7.4, $150 \mathrm{~mm} \mathrm{NaCl}, 1 \mathrm{~mm}$ EDTA, 1\% TritonX-100, $0.5 \%$ NP-40, $1 \mathrm{~mm}$ PI, $1 \mathrm{~mm}$ DTT, $1 \mathrm{~mm}$ PMSF and placed on ice for $1 \mathrm{~h}$. Cell lysates ( $50 \mu \mathrm{g} /$ lane) were separated by SDSPAGE and transferred to PVDF (polyvinylidene difluoride) membrane. Blots were blocked with 5\% skim milk in PBS containing $0.05 \%$ Tween 20 for $1 \mathrm{~h}$ at $25^{\circ} \mathrm{C}$, then incubated with primary antibodies, followed by incubation with antirabbit horseradish peroxidase-conjugated IgG and visualized with enhanced chemiluminescence. Primary antibodies used were as follows: rabbit anti-caspase-3, 1:1000; rabbit antiPARP, $1: 1000$; mouse anti-FASN, $1: 250$; rabbit anti-Gapdh, $1: 2000$.

DNA Fragmentation Assay Cells were plated at $2 \times 10^{5}$ cells $/ \mathrm{ml}$ in 12-well plates and added amentoflavone for $48 \mathrm{~h}$. Both attached and detached cells were collected and lysed in $100 \mathrm{~mm}$ Tris (pH 8.0), $20 \mathrm{~mm}$ EDTA, 0.8\% $N$-laurylsarcosine sodium salt on ice. The lysates were incubated with $0.2 \mathrm{mg} / \mathrm{ml} \mathrm{RNase} \mathrm{A}$ for $1 \mathrm{~h}$ at $37^{\circ} \mathrm{C}$, then with $4 \mathrm{mg} / \mathrm{ml}$ Proteinase $\mathrm{K}$ for $\mathrm{O} / \mathrm{N}$ at $50^{\circ} \mathrm{C}$. The $\mathrm{DNA}$ was precipitated by adding an equal volume of isopropanol, and loaded on to $2 \%$ agarose gels and electrophoresed in 1X TBE buffer for $3 \mathrm{~h}$. Gels were photographed under UV light.

Cell Proliferation and Viability Assay Cells were seeded at $5 \times 10^{3}$ cells $/ \mathrm{ml}$ in 96 -well microplates and allowed to attach for $24 \mathrm{~h}$. Amentoflavone was added to the medium at various concentrations up to $100 \mu \mathrm{M}$. After treatment, cell cytotoxicity and/or proliferation was assessed by Cell Counting Kit8 (CCK8). Briefly, highly water-soluble tetrazolium salt, WST-8[2-(2-methoxy-4-nitrophenyl)-3-(4-nitrophenyl)5-(2,4-disulfophenyl)-2H-tetrazolium, monosodium salt], produced an orange colored water-soluble product, formazan. The amount of formazan dye generated by dehydrogenases in cells was directly proportional to the number of living cells. CCK8 $(10 \mu \mathrm{l})$ was added to each well and incubated for $3 \mathrm{~h}$ at $37^{\circ} \mathrm{C}$, then cell proliferation and cytotoxicity were assessed by measuring the absorbance at $450 \mathrm{~nm}$ using microplate reader. Three replicated wells were used for each experimental condition.

\section{RESULTS}

Amentoflavone Inhibited FASN Activity in Vitro The pharmacological inhibitors of FASN are receiving increasing attention as suggested by recent reports indicating that FSAN may represent a molecular bridge connecting obesity and cancer. ${ }^{20)}$ Previously, some natural flavonoids were found to inhibit FASN, with $\mathrm{IC}_{50}$ values ranging from 2 to $112 \mu \mathrm{M},{ }^{21)}$ but no further reports of natural flavonoids in fatty acid metabolism were published. Therefore we screened medicinal plants using an FASN activity assay and selected $S$. tamariscina $\left(\mathrm{IC}_{50}\right.$ value of $\left.72.15 \pm 0.34 \mu \mathrm{g} / \mathrm{ml}\right)$ for this particular study. A bioactive compound was extracted and isolated from the plant and identified amentoflavone, a naturally occurring biflavonoid of $\geq 95 \%$ purity (Fig. 1). After purification, we examined the effect of amentoflavone on FASN ac- 


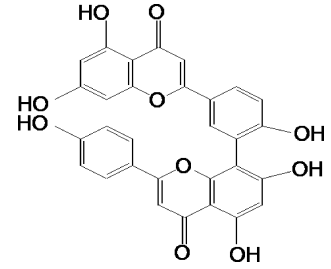

Fig. 1. Chemical Structure of Amentoflavone Isolated from S. tamariscina

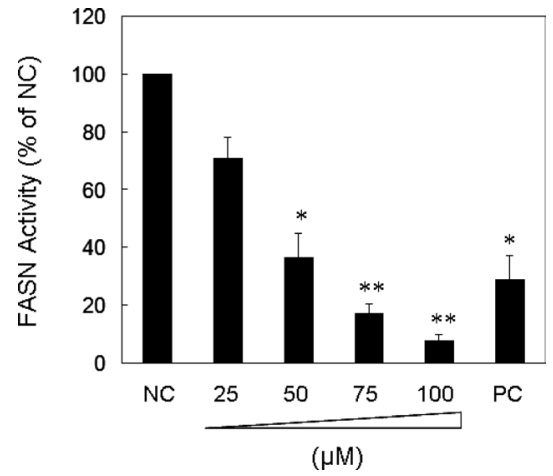

Fig. 2. In Vitro FASN Activity by Amentoflavone

The reaction buffer (200 mm K-P buffer, pH 7.0, $2.5 \mathrm{~mm}$ DTT, 2 mм EDTA, $1 \mathrm{mg} / \mathrm{ml}$ BSA) was incubated with FASN enzyme and amentoflavone $(0-100 \mu \mathrm{M})$ for $1 \mathrm{~h}$, and incubated with $0.25 \mathrm{~mm}$ NADPH, $4 \mu \mathrm{M}$ Malonyl-CoA, $0.2 \mu \mathrm{M}$ Acetyl-CoA, $0.02 \mu \mathrm{Ci}$ $\left[{ }^{3} \mathrm{H}\right]$ acetyl-CoA for $10 \mathrm{~min}$ at $37^{\circ} \mathrm{C}$. After the reaction termination with $60 \% \mathrm{HClO}$ hexane-soluble fraction was determined by scintillation counter. Cerulenin $(50 \mu \mathrm{M})$ was used as a postitive control (PC). Each data point represents the mean \pm S.E.M. for three independent experiments. $* p<0.01$ and $* * p<0.001 v s$. negative control.

tivity in vitro. As shown in Fig. 2, amentoflavone showed a dose-dependent decrease and significant inhibition of FASN activity by $63.7 \%$ at $50 \mu \mathrm{M}, 83 \%$ at $75 \mu \mathrm{M}$ and $92.4 \%$ at $100 \mu \mathrm{M}$. The positive control, cerulenin, decreased FASN activity by $71.4 \%$ at a dose of $50 \mu \mathrm{M}$. These results indicated that amentoflavone may have potently blocked FASN activity and may thus be a potential FASN inhibitor.

Amentoflavone Inhibited Fatty Acid Synthesis in FASN-Overexpressed SK-BR-3 Breast Cancer Cells To determine FASN expression in SK-BR-3 breast cancer and NIH-3T3 normal cells, Western blotting was performed. This study showed that SK-BR-3 cells were FASN-positive with higher FASN expression than NIH-3T3 (Fig. 3A). To characterize the effect of amentoflavone on fatty acid synthesis, FASN-overexpressed SK-BR-3 cells were cultured, and incubated for $24 \mathrm{~h}$ in media supplemented with amentoflavone at varying concentrations. Fatty acid synthesis activity was measured by the incorporation of $\left[{ }^{3} \mathrm{H}\right]$ acetyl-CoA into total cellular lipids. The results showed that amentoflavone decreased fatty acid synthesis by $12.7 \%$ at $25 \mu \mathrm{M}, 54.4 \%$ at $50 \mu \mathrm{M}, 77.4 \%$ at $75 \mu \mathrm{M}$ and $83 \%$ at $100 \mu \mathrm{M}$, compared to the negative control (NC) (Fig. 3B). Meanwhile, cerulenin as the positive control suppressed fatty acid synthesis by $57 \%$ at $50 \mu \mathrm{M}$. These findings indicated that amentoflavone was a novel FASN-inhibitor that was effective on the cellular level.

Activation of Caspase-3 was Involved in Amentoflavone-Induced Apoptosis Several reports indicated a potential role for FASN as a therapeutic target in a wide range of cancers. Chemical FASN blockers such as cerulenin and C75, formerly characterized as antitumor cytotoxic agents, may act as therapeutic moieties in breast cancers. ${ }^{22)}$ We thus
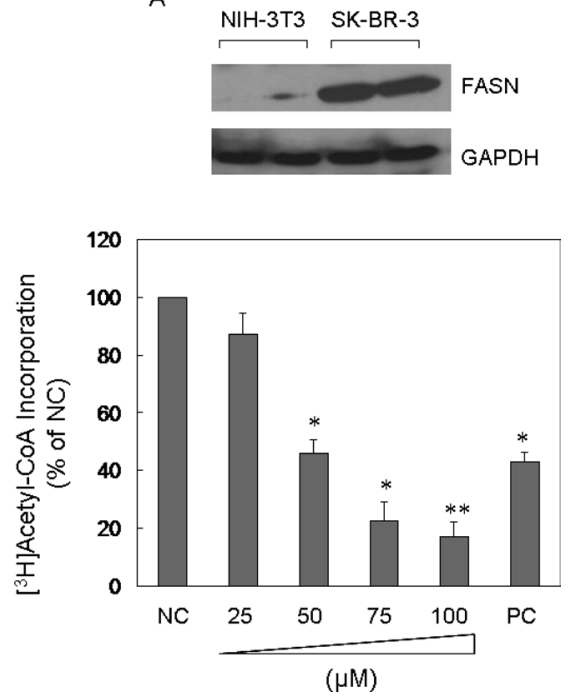

Fig. 3. FASN-Overexpression and Fatty Acid Synthesis Effects of Amentoflavone in SK-BR-3 Cells

(A) FASN-expression was observed by Western blotting in SK-BR-3 breast cancer and NIH-3T3 normal cells. (B) SK-BR-3 cells were treated for $24 \mathrm{~h}$ with amentoflavone $(0$ to $100 \mu \mathrm{M})$, then incubated with $\left[{ }^{3} \mathrm{H}\right]$ acetyl-CoA $(2 \mu \mathrm{Ci})$ for $4 \mathrm{~h}$. And cells were collected, washed, recounted, then cellular lipids were extracted and quantified relative to vehicle-treated control. Cerulenin $(50 \mu \mathrm{M})$ was used as a postitive control (PC). Each data point represents the mean \pm S.E.M. for three independent experiments. $* p<0.05$ and $* * p<0.001 v s$. negative control.

examined whether the novel FASN inhibitor amentoflavone, could deplete FASN-expression and influence apoptosis and cell death through activation of caspase-3 and PARP using SK-BR-3 breast cancer cells. First we observed a decrease of FASN expression by amentoflavone, although the magnitude of depletion was not very prominent (Fig. 4A). Caspase-3 is a critical executioner of apoptosis, as it is either partially or totally responsible for the proteolytic cleavage of many key proteins such as the nuclear enzyme PARP, which plays an important role in condensation and degradation of chromatin of cells going through apoptosis. Activation of caspase-3 requires the proteolytic processing of its inactive zymogen $(34 \mathrm{kDa})$ into activated p18 and p20 fragments. PARP $(116 \mathrm{kDa})$ is also cleaved into a p85 fragment by induction of apoptosis. The results of the present study showed that amentoflavone was able to initiate activation of caspase-3-dependent apoptosis and PARP cleavage at $75 \mu \mathrm{M}$. This was followed by a dramatic activation after treatment with $100 \mu \mathrm{M}$ of amentoflavone (Fig. 4A). To further confirm the involvement of caspases, including caspase-3, in the apoptosis induced by amentoflavone, SK-BR-3 cells were pretreated with the pan-caspase inhibitor Z-VAD-FMK for $2 \mathrm{~h}$, followed by amentoflavone treatment. The caspase inhibitor completely rescued the cells from the PARP cleavage induced by amentoflavone (Fig. 4B). No effects of amentoflavone on caspase-3-dependent apoptosis and PARP cleavage in FASNnonexpressed NIH-3T3 normal cells (Fig. 4C). These findings underlined the capacity of amentoflavone to stimulate caspase-3-induced apoptosis on FASN-expressed SK-BR-3 cells.

Amentoflavone Stimulated DNA Fragmentation To further confirm whether apoptosis was induced by amentoflavone in SK-BR-3 cells, electrophoretic analysis of fragmented DNA was conducted. SK-BR-3 cells were treated 


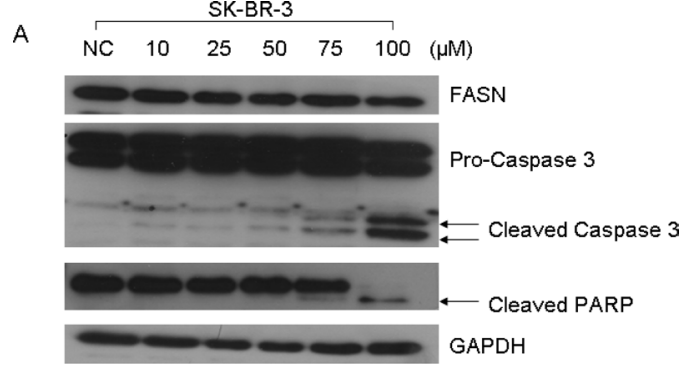

B

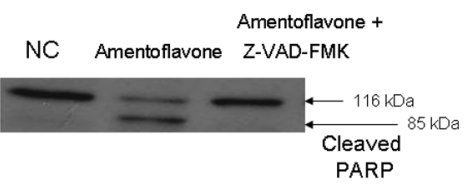

C

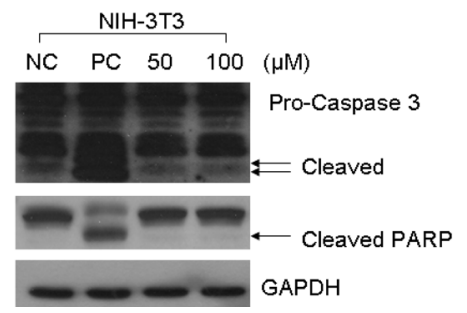

Fig. 4. Apoptosis Induced by Amentoflavone in FASN-Overexpressed SK-BR-3 Cells

(A) FASN expression and cleavages of apoptosis-related proteins were measured by Western blot analysis after amentoflavone treatment $(0-100 \mu \mathrm{M})$ in SK-BR-3 cells. Equal amount of cell lysates $(50 \mu \mathrm{g})$ were resolved by SDS-PAGE, transferred to PVDF membrane, and probed with specific actibodies, anti-FASN, anti-caspase-3, anti-PARP and anti-Gapdh antibody to serve as control for loading of protein level. (B) Pan-caspase inhibitor Z-VAD-FMK $(100 \mu \mathrm{M})$ was pre-treated for $2 \mathrm{~h}$ and added amentoflavone $(100 \mu \mathrm{M})$ in SK-BR-3 cells, and PARP cleavage was measured by Western blot analysis. (C) The cleavages of caspase-3 and PARP were measured by Western blot analysis after amentoflavone treatment $(50,100 \mu \mathrm{M})$ in NIH-3T3 normal cells.

with various concentrations of amentoflavone or a positive control $(10 \mu \mathrm{M})$, and then low-molecular weight DNA fragments were extracted and visualized by electrophoresis. The electrophoretic separation of the fragments yielded a ladder-like pattern observed after treatment of cells with $100 \mu \mathrm{M}$ amentoflavone, but not $50 \mu \mathrm{M}$ (Fig. 5A). No DNA fragment was observed with a concentration of $100 \mu \mathrm{M}$ of amentoflavone in FASN-nonexpressed NIH-3T3 normal cells (Fig. 5B). The results supported the postulation that amentoflavone was able to activate apoptosis in FASN-expressed SK-BR-3 cells.

Amentoflavone Decreased Proliferation of Breast Cancer Cells The antiproliferative effect of amentoflavone on SK-BR-3 cells was examined by exposing the cells to amentoflavone for 12, 24 and $48 \mathrm{~h}$. Following treatment with amentoflavone, cell growth was inhibited by $2.7 \%$ after $12 \mathrm{~h}$ and $23 \%$ after $24 \mathrm{~h}$. Cell growth was dramatically suppressed by $80 \%$ after $48 \mathrm{~h}$ post amentoflavone treatment, when compared to the negative control $(0 \mathrm{~h})$ (Fig. 6A). After treatment with amentoflavone for $48 \mathrm{~h}$, a dose-dependent inhibition of cell viability was observed by $3.7 \%$ at $25 \mu \mathrm{M}, 24 \%$ at $50 \mu \mathrm{M}$, $67 \%$ at $75 \mu \mathrm{M}$ and $82.7 \%$ at $100 \mu \mathrm{M}$ (Fig. 6B). No significant effects of amentoflavone on cell proliferation in FASN-non-
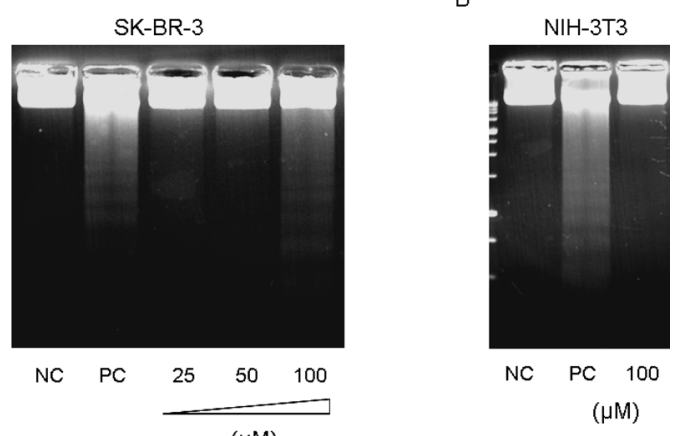

Fig. 5. Fragmentations of Genomic DNA by Amentoflavone in SK-BR-3 and NIH-3T3 Cells

SK-BR-3 (A) and NIH-3T3 Cells (B) were treated for $24 \mathrm{~h}$ with the indicated concentrations of amentoflavone. Fragmented DNA was extracted and analyzed on $2 \%$ agarose gel. Camptothecin $(10 \mu \mathrm{M})$ was used as a positive control (PC).

A
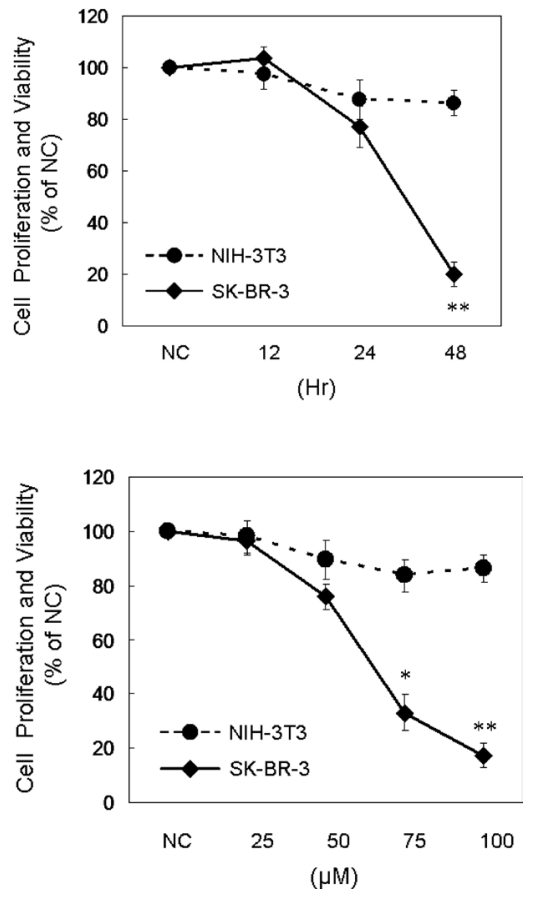

Fig. 6. Time- and Dose-Dependent Effects of Amentoflavone on Cell Proliferation and Viability in SK-BR-3 and NIH-3T3 Cells

(A) Cells were treated with $100 \mu \mathrm{m}$ amentoflavone for 12,24 , and $48 \mathrm{~h}$. Cell proliferation and viability were determined by CCK 8 assay. The percentage of cell viability was calculated as a ratio of $\mathrm{A} 450 \mathrm{~nm}$ of amentoflavone treated cells and untreated cells Each data point represents the mean \pm S.E.M. for three independent experiments ** $p<0.001 v s$. negative control. (B) Cells were treated with $25,50,75$ and $100 \mu \mathrm{M}$ amentoflavone for $48 \mathrm{~h}$. Each data point represents the mean \pm S.E.M. for three independent experiments. $* p<0.01$ and $* * p<0.001 v s$. negative control.

expressed NIH-3T3 normal cells were observed in both doseand time-dependent manners. To confirm that growth inhibition was due to FASN inhibition, we investigated the effects of amentoflavone on cell proliferation in another FASN-overexpressed cell line, LNCaP human prostate cancer cells. Similar results were obtained (data not shown). The results showed that amentoflavone might be an antiproliferative agent particularly in FASN-expressed cancer cells. 


\section{DISCUSSION}

FASN, capable of de novo fatty acid synthesis, is highly expressed in most aggressive human breast carcinomas. The expression of FASN is an indicator of poor prognosis in breast cancer and is found to be elevated in the blood of cancer patients. Inhibition of FASN-expression is selectively cytotoxic to human breast cancer cells. ${ }^{3)}$ Why inhibition of fatty acid synthesis selectively kills cancer cells remains unclear. Previous studies explored the inhibition of fatty acid synthesis through FASN-inhibition, which induced apoptosis under a number of modulated pathways. ${ }^{13,25)}$ For instance, nonfunctioning p53 proteins by pharmacological inhibition and small interfering RNA (siRNA)-mediated gene silencing, HER2/neu regulation, p-Akt downregulation, ${ }^{26-29)}$ and recently endoplasmic reticulum stress response were reported. ${ }^{4)}$ Thus, high levels of FASN expression and its association with tumor prognosis, led to the exploration of FASN as a drug target for cancer therapy. Furthermore, the FASN-overexpressed aggressiveness of cancers is partly attributed to its resistance to chemotherapeutic agent-induced apoptosis. To overcome the apoptotic resistance offered by breast cancer cells, novel agents that possess high therapeutic potential with less systemic toxicity are being evaluated worldwide.

Many diet-based polyphenolic agents with high anticancer efficacy and no or acceptable levels of toxicity to normal tissues have been suggested as possible candidates for use by breast cancer patients. Due to low immunogenicity and high stability, natural agents that induce apoptosis may provide the opportunity for minimal acquired drug resistance, decreased mutagenesis, and reduced toxicity. ${ }^{30}$ ) In epidemiological studies, phytochemicals supported the potential role in the prevention and treatment of breast cancer. ${ }^{31)}$ From an overall perspective, present and previous studies support the postulation that FASN-inhibition lowers chemotherapeutic resistance and retards breast cancer cell growth effectively, with apoptosis playing an important role to their demise. Based on these suggestions, we screened a variety of medicinal plants and selected $S$. tamariscina, and its major constituent amentoflavone, a natural biflavonoid consisting of an apigenin dimmer, and confirmed the inhibition of lipogenic activity in FASN-overexpressed SK-BR-3 breast cancer cells (Figs. 2, 3).

Furthermore our results revealed that amentoflavone induced apoptosis in SK-BR-3 breast cancer cells. Apoptosis is a major form of cell death and has become a novel target for cancer chemoprevention and chemotherapy in recent years. ${ }^{32)}$ Since amentoflavone has been widely used for advanced cancer treatment, ${ }^{18)}$ and FASN- inhibitors have been shown to be involved in apoptosis through caspase-3 activation and PARP cleavage, FASN-inhibitors may possess great potential to be used in the stimulation of apoptosis particularly in aggressive breast cancer cells. ${ }^{33}$ ) Specific FASN-inhibitors, including cerulenin, $\mathrm{C} 75$, triclosan and orlistat, have been reported to possess anti-cancer activity. ${ }^{8)}$ Like other FASNinhibitors, which were suggested to stimulate apoptosis in MCF7, MDA-MB-231 and SK-BR-3 breast cancer cells, ${ }^{34)}$ amentoflavone also induced caspase-3-dependent apoptosis and subsequently activated PARP cleavage and DNA fragmentation in SK-BR-3 cells (Figs. 4, 5). Apart from caspasedependent apoptosis, it seems that FASN-inhibitors exert their growth-inhibitory effect via $\mathrm{p} 21$ and cell cycle arrest. ${ }^{33)}$ Inhibition of FASN activity by amentoflavone caused inhibition of SK-BR-3 breast cancer cell growth in our study (Fig. $6)$.

In conclusion, we showed that amentoflavone isolated from S. tamariscina exerted FASN-inhibitory activity, induced caspase-dependent apoptosis, and decreased the proliferation of FASN-overexpressed SK-BR-3 breast cancer cells. The reduction of lipid synthesis by amentoflavone stimulated major apoptosis indicators, including caspase-3 and PARP activations as well as DNA fragmentation, dramatically decreasing the growth of breast cancer cells without toxicity to normal cells. Therefore, we propose that amentoflavone, a novel FASN-inhibitor, has the potential to delay cancer progression and induce apoptosis, properties essential for cancer therapy particularly in aggressive breast carcinoma. We recommend that further studies be conducted to investigate the molecular mechanism of action involved in the link between fatty acid metabolism and apoptosis.

\section{REFERENCES}

1) Wakil S. J., Biochemistry, 28, $4523-4530$ (1989).

2) Baron A., Migita T., Tang D., Loda M., J. Cell. Biochem., 91, 47-53 (2004).

3) Kuhajda F. P., Cancer Res., 66, 5977-5980 (2006).

4) Little J. L., Wheeler F. B., Fels D. R., Koumenis C., Kridel S. J., Cancer Res., 67, 1262-1269 (2007).

5) Kuhajda F. P., Nutrition, 16, 202-208 (2000).

6) Kuhajda F. P., Jenner K., Wood F. D., Hennigar R. A., Jacobs L. B. Dick J. D., Pasternack G. R., Proc. Natl. Acad. Sci. U.S.A., 91, 63796383 (1994).

7) Kuhajda F. P., Pizer E. S., Li J. N., Mani N. S., Frehywot G. L., Townsend C. A., Proc. Natl. Acad. Sci. U.S.A., 97, 3450-3454 (2000).

8) Kridel S. J., Axelrod F., Rozenkrantz N., Smith J. W., Cancer Res., 64, 2070-2075 (2004)

9) Knowles L. M., Axelrod F., Browne C. D., Smith J. W., J. Biol. Chem., 279, 30540-30545 (2004).

10) Alo P. L., Visca P., Marci A., Mangoni A., Botti C., Di Tondo U., Cancer, 77, 474-482 (1996)

11) Jensen V., Ladekarl M., Holm-Nielsen P., Melsen F., Soerensen F. B., J Pathol., 176, 343-352 (1995).

12) Pizer E. S., Jackisch C., Wood F. D., Pasternack G. R., Davidson N. E., Kuhajda F. P., Cancer Res., 56, 2745-2747 (1996).

13) Pizer E. S., Thupari J., Han W. F., Pinn M. L., Chrest F. J., Frehywot G L., Townsend C. A., Kuhajda F. P., Cancer Res., 60, 213-218 (2000).

14) Yim D., Singh R. P., Agarwal C., Lee S., Chi H., Agarwal R., Cancer Res., 65, 1035-1044 (2005).

15) Mora A., Payá M., Ríos J. L., Alcaraz M. J., Biochem. Pharmacol., 40, 793-797 (1990)

16) Kang D. G., Yin M. H., Oh H., Lee D. H., Lee H. S., Planta Med., 70, $718-722$ (2004).

17) Lin Y. M., Anderson H., Flavin M. T., Pai Y. H., Mata-Greenwood E., Pengsuparp T., Pezzuto J. M., Schinazi R. F., Hughes S. H., Chen F. C., J. Nat. Prod., 60, 884-8 (1997).

18) Guruvayoorappan C., Kuttan G., Biochemistry (Mosc.), 73, 209-218 (2008).

19) Tian W. X., Hsu R. Y., Wang Y. S., J. Biol. Chem., 260, 11375-11387 (1985).

20) Menendez J. A., Lupu R., Colomer R., Mol. Genet. Metab., 84, 293295 (2005)

21) Li B. H., Tian W. X., J. Biochem., 135, 85-91 (2004).

22) Wang Y. Y., Kuhajda F. P., Li J., Finch T. T., Cheng P., Koh C., Li T., Sokoll L. J., Chan D. W., J. Exp. Ther. Oncol., 4, 101-110 (2004).

23) Pilat M. J., Kamradt J. M., Pienta K. J., Cancer Metastasis Rev., 17, 373-381 (1999).

24) Fernandes-Alnemri T., Litwack G., Alnemri E. S., J. Biol. Chem., 269, $30761-30764$ (1994) 
25) Zhou W., Simpson P. J., McFadden J. M., Townsend C. A., Medghalchi S. M., Vadlamudi A., Pinn M. L., Ronnett G. V., Kuhajda F. P., Cancer Res., 63, 7330-7337 (2003)

26) Li J. N., Gorospe M., Chrest F. J., Kumaravel T. S., Evans M. K., Han W. F., Pizer E. S., Cancer Res., 61, 1493-1499 (2001).

27) Menendez J. A., Lupu R., Int. J. Mol. Med., 15, 33-40 (2005).

28) Menendez J. A., Vellon L., Mehmi I., Oza B. P., Ropero S., Colomer R., Lupu R., Proc. Natl. Acad. Sci. U.S.A., 101, 10715-10720 (2004).

29) Wang H. Q., Altomare D. A., Skele K. L., Poulikakos P. I., Kuhajda F. P., Di Cristofano A., Testa J. R., Oncogene, 24, 3574-3582 (2005).
30) Saleem M., Kweon M. H., Yun J. M., Adhami V. M., Khan N., Syed D. N., Mukhtar H., Cancer Res., 65, 11203-11213 (2005).

31) Wang Y., Ye L., Leung L. K., Toxicology, 248, 130-135 (2008).

32) Liu X., Yue P., Zhou Z., Khuri F. R., Sun S. Y., J. Natl. Cancer Inst., 96, 1769-1780 (2004).

33) Ho T. S., Ho Y. P., Wong W. Y., Chi-Ming Chiu L., Wong Y. S., EngChoon Ooi V., Biomed. Pharmacother, 61, 578-587 (2007).

34) Heiligtag S. J., Bredehorst R., David K. A., Cell. Death Differ, 9, $1017-1025$ (2002). 